\title{
Detection of carotid artery plaques in the dental setting*
}

\author{
Alexandre Rezende Vieira", Lisa Marie Babb \\ Department of Oral Biology, School of Dental Medicine, University of Pittsburgh, Pittsburgh, USA \\ Email: \#arv11@pitt.edu
}

Received 29 February 2012; revised 26 March 2012; accepted 7 April 2012

\begin{abstract}
It has been suggested that atherosclerotic plaques can be visualized in a panoramic radiograph but this tool is not reliable for systematic screening of patients due to its low sensitivity. This study investigated the potential role for dental practitioners in using an ultrasound machine to detect carotid artery plaques. 100 subjects completed a survey concerning theiratherosclerosis risk factors. Bilateral images and digital video clips of the carotid arteries of each person were obtained with a portable high-resolution ultrasound device equipped with an L38/10-5 MHz linear-array transducer. Images were subsequently interpreted. We found images suggestive of atherosclerotic plaque build-up in two Caucasian females: one 59 year-old with risk factors including: past smoker, high cholesterol, and family history of atherosclerosis and one 54 year-old with risk factors including: past smoker, overweight, and reported no physical activity. Both individuals were referred to their primary care providers for further assessments. $2 \%$ of the patient adult population may have carotid blockages detectable by ultrasound. The frequency can be as high as $5.5 \%$ in individuals older than 50 . In the dental setting, ultrasound imaging can be used to capture bilateral pictures of the carotid arteries. With these images, at-risk patients may be screened for atherosclerotic plaques that could ultimately form a strokecausing embolus. The dentist is particularly well suited for this role because he/she sees patients every six months while a physician can go years without seeing his/her patients.
\end{abstract}

Keywords: Cardiovascular Diseases; Dental Education; Atherosclerosis

\section{INTRODUCTION}

For a number of years, the method in which the oral condition affects systemic health has become a key focus of

\footnotetext{
"The authors have no conflict of interest to declare.

"Corresponding author.
}

study. One aspect of this issue discusses how the dental professional can play a major role in the prevention of major chronic systemic illnesses and their complications.

According to the Centers for Disease Control and Prevention (CDC; http://www.cdc.gov/heartdisease/), cardiovascular diseases are the number one cause of mortality in the US and a major cause of disability. For a number of years, panoramic radiographs have been suggested as a tool for detecting carotid artery calcificationsbecause these anatomic structures are potentially within the visual field of these radiographs [1-11]. However, data suggest panoramic radiographs have low sensitivity and positive predictive value and are considered unreliable for detecting carotid artery calcification [12-15]. In the beginning of the 1980s, ultrasound measurement of carotid arteries was introduced and this assessment is currently available in clinical practice [16].

The future of the dental profession has been discussed and some dental educators now advocate an oral physiccian model as the desired direction for dentistry, with expanded training in systemic disease pathophysiology and a practice scope that extends beyond exclusive focus on the teeth and supporting structures [17].

Head and neck structures are part of the scope of the dental profession and evaluation of these structures could play a role in enhancing patient care, as it has been suggested by the careful evaluation of panoramic radiographs [1-11]. In this work, we present data on a pilot study that aimed to evaluate the feasibility of a dentist to use an ultrasound to assess the presence of carotid artery calcifications in the dental setting and discuss the potential of this procedure being incorporated into the scope of the dental profession.

\section{METHODOLOGY}

This pilot study included the bilateral assessment of the carotid arteries of 100 subjects 18 years of age or older performed between October and December 2008. The goal was to evaluate the feasibility of using at the dental chair a portable high-resolution ultrasound device equiped with an L38/10-5 MHz linear-array transducer model Titan (SonoSite Inc., Bothell, USA) and interpreting the 
images. This protocol was approved by the University of Pittsburgh Institutional Review Board (protocol \#PRO08080413) and all subjects provided written informed consent prior to participation in the study. We recruited a convenient sample of adults that work and study at the University of Pittsburgh School of Dental Medicine. Age, gender, and self-reported ethnic background of the subjects was recorded and participants answered a brief questionnaire that included questions about their height, weight, blood pressure, family history of atherosclerosis, smoking habits, history of diabetes, high cholesterol, and exercise habits. While seated in a dental chair, the operator (L.M.B.), supervised by A.R.V. scanned each subject's carotid arteries on both sides of the neck. Six images (three on each side captured at different levels of the common carotid artery) and two 20-second movies (one on each side, scanning the common carotid) were recorded for each subject both cross-sectional and longitudinally. Images and movies were evaluated within 48 hours and were made available to subjects upon request. In the case of the identification of possible carotid artery calcifications, the subjects received a letter explaining the findings and suggesting them to visit their primary care provider for further evaluations.

\section{RESULTS}

From the 100 subjects studied, 64 were females and 36 males. According to them, 80 were Caucasian, six African American, six Asian, two Hispanic, and six did not answer the question. The average age of the study population was 42 years (ranging from 18 to 80 years) and 36 subjects were older than 50 . Based on body-mass index determinations, 53 individuals were healthy, 29 overweight, 11 obese, and one extremely obese. Fourteen individuals reported high cholesterol levels, two diabetes, six were current smokers (averaging 0.5 pack/day), and 16 were past smokers (averagingone pack/day). All subjects reported blood pressure within normal limits, some controlled by medication. We found images suggestive of atherosclerotic plaque build-up in two Caucasian females: one 59 year-old with risk factors including: past smoker, high cholesterol, and family history of atherosclerosis, and one 54 year-old with risk factors including: past smoker, overweight, and reported no physical activity. Both individuals were referred to their primary care providers for further assessments and their physicians confirmed the findings by an independent ultrasound done by an experienced technician. Both subjects were already under treatment for high blood pressure and/or diet counseling to lower cholesterol levels and smoke cessation therapy. Figures $\mathbf{1}$ and $\mathbf{2}$ exemplify the findings of an unaltered carotid artery in contrast with one with image suggesting partial carotid artery plaque build-up.

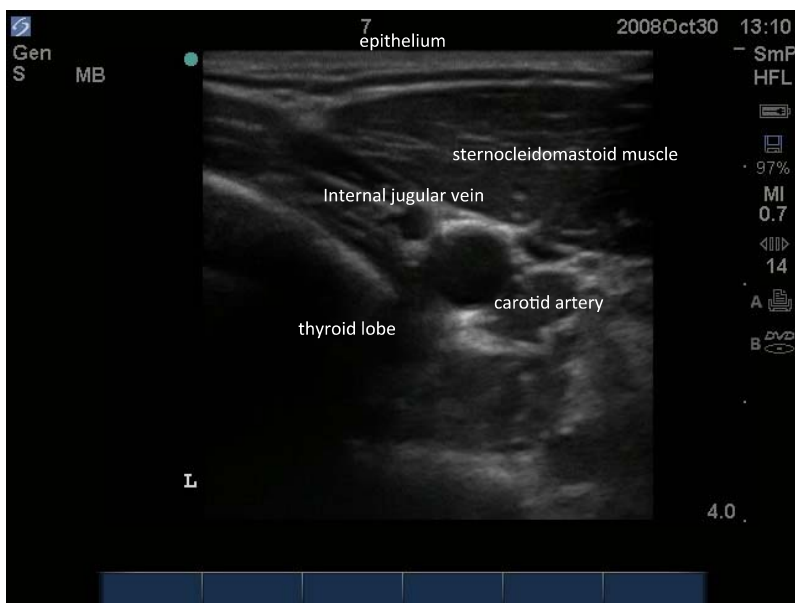

Figure 1. Carotid artery without alterations.

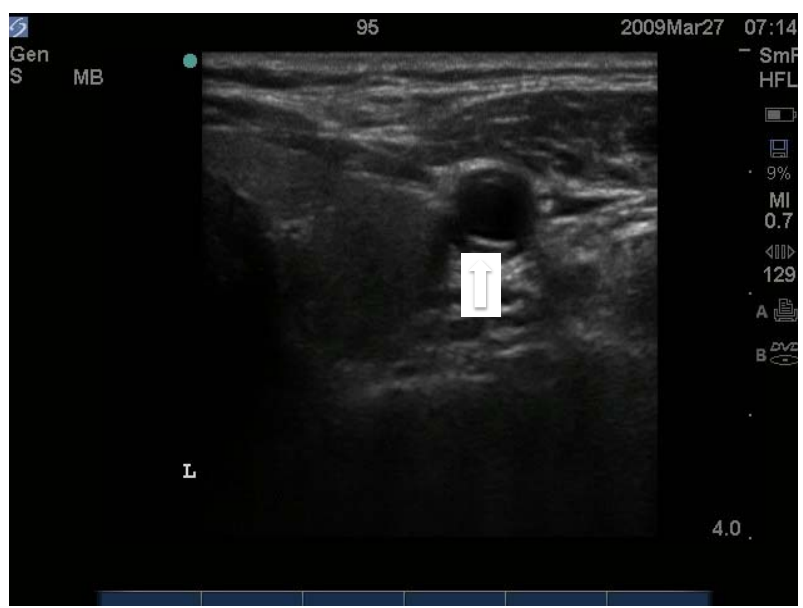

Figure 2. Carotid artery showing deposits the bottom of the artery lumen (arrow).

No subjects reported to have had any discomfort related to the procedures performed.

\section{DISCUSSION}

Our preliminary results suggest the dental professional can identify carotid artery plaque build-ups under the ultrasound in the dental setting. The frequency of our findings was $2 \%$ (2 in 100), suggesting that this is the frequency of the patient adult population that may have carotid blockages detectable by ultrasound. The frequency can be as high as $5.5 \%$ in individuals older than 50 and this figure is very similar to a previous study that suggested carotid artery calcifications are found in up to $5 \%$ of older patients [18]. In addition, since we did not focus on the proximal component of internal carotid artery, an untold number of atherosclerotic lesions may have been missed.

There are a number of interesting aspects to be discussed. Of greatest concern is the appropriateness of dentists "de novo" screening patients for atherosclerotic 
disease. Many physicians might construe this act as practicing medicine and beyond the scope of dental licensure. Similarly, most dentists might likewise question this practice since it bears no resemblance to identifying calcific lesions of this nature on panoramic radiographs obtained during the normal course of diagnosing and treating oral diseases. Will physicians perceive this kind of approach as an encroachment on territory? No doubt some of these same criticisms were leveled against those dentists who first took patients' blood pressures. The past decade has been marked by debate about the future directions of the profession [17], and this kind of procedure can be advocated as an additional resource to enhance patient care and disease prevention.

A second issue relates to the dental professional performing this assessment. Should the general dentist performthis procedure or someone with specialty training in radiology and imaging should be primarily performing this type of exam? The argument in favor of the latter may be that this professional will be better equipped to identify other alterations in the neck structures, such as cysts or thyroid malformations. Furthermore, ultrasound equipment, even the portable model used in this study, is still costly and probably better suited for a business focused on imaging.

Finally, the most interesting finding is that the dentist can still play a major role in regards to prevention of cardiovascular diseases. It is recognized that patients visit the dentist every six months meaning that, in theory, dentists see their patients more often than primary care providers. The two subjects in whom we detected carotid artery build-ups have several risk factors for cardiovascular diseases based on the information we collected. Dentists are in a unique position to discuss such risks factors of disease that may negatively impact individual quality of life. Therefore, we argue that we should not indicate that all dentists should start performing neck ultrasounds in their offices (although our data suggest carotid artery build-ups can be detected in the dental setting by this technique), but dentists have the potential to impact their patients' risks for cardiovascular diseases. The current discussion in dental education includes the argument that the bulk of the dental school curriculum is devoted to tooth restoration or replacement techniques, although the need for these procedures has declined. Therefore, dental training should expand to a deeper understanding of systemic disease and the practice scope should expand beyond exclusive focus on teeth and supporting structures [17].

After this pilot project, this work should be expanded to further evaluate the feasibility of a dentist to use an ultrasound to assess the presence of carotid artery plaques in the dental setting, we aim to recruit $20-30$ dentists and ask them to use the ultrasound on subjects with and withoutcarotid artery plaques. There they should evaluate:

- The ease of use;

- The time needed for training;

- Amount of true positives/true negatives and false positives/false negatives.

\section{CONCLUSION}

In the dental setting, ultrasound imaging can be used to capture bilateral pictures of the carotid arteries. Cardiovascular risks can be identified while gathering medical history, giving the dentist the potential to advocate prevention of these diseases with their patients and refer them to primary care physicians for early management.

\section{ACKNOWLEDGEMENTS}

The authors thank study subjects for their enthusiastic participation. This project was made possible by Grant Number UL1 RR024153 from the National Center for Research Resources (NCRR), a component of the National Institutes of Health (NIH), and NIH Roadmap for Medical Research. Its contents are solely the responsibility of the authors and do not necessarily represent the official view of NCRR or NIH.

\section{REFERENCES}

[1] Friedlander, A.H., Manesh, F. and Wasterlain, C.G. (1994) Prevalence of detectable carotid artery calcifications on panoramic radiographs of recent stroke victims. Oral Surgery Oral Medicine Oral Pathology, 77, 669-673. doi:10.1016/0030-4220(94)90332-8

[2] Carter, L.C., Tsimidis, K. and Fabiano, J. (1998) Carotid calcifications on panoramic radiography identify an asymptomatic male patient at risk for stroke. A case report. Oral Surgery Oral Medicine Oral Pathology Oral Radiology \& Endodontics, 85, 119-122. doi:10.1016/S1079-2104(98)90409-7

[3] Almog, D.M., Illig, K.A., Khin, M. and Green, R.M. (2000) Unrecognized carotid artery stenosis discovered by calcifications on a panoramic radiograph. Journal of American Dental Association, 131, 1593-1597.

[4] Ohba, T., Takata, Y., Ansai, T., Morimoto, Y., Tanaka, T., Kito, S., Awano, S., Akifusa, S. and Takehara, T. (2003) Evaluation of calcified carotid artery atheromas detected by panoramic radiograph among 80-year-olds. Oral Surgery Oral Medicine Oral Pathology Oral Radiology \& Endodontics, 96, 647-650. doi:10.1016/j.tripleo.2003.07.001

[5] Almog, D.M., Illig, K.A., Elad, S., Romano, P.R. and Carter, L.C. (2005) Supplementary role of panoramic radiographs in the medical surveillance of a patient at risk for stroke. Compendium of Continuing Educationin Dentistry, 26, 369-372.

[6] Bayram, B., Uckan, S., Acikgoz, A., Müderrisoglu, H. and Aydinalp, A. (2006) Digital panoramic radiography: A reliable method to diagnose carotid artery atheromas? 
Dentomaxillofacial Radiology, 35, 266-270. doi:10.1259/dmfr/50195822

[7] Kumagai, M., Yamagishi, T., Fukui, N. and Chiba, M. (2007) Long-term cigarette smoking increases the prevalence of carotid artery calcification seen on panoramic dental radiographs in male patients. Tohoku Journal of Experimental Medicine, 212, 21-25. doi:10.1620/tjem.212.21

[8] Ramesh, A. and Pabla, T. (2007) Panoramic radiographs: A screening tool for calcified carotid atheromatous plaque. Journal of Massachusetts Dental Society, 56, 20-21.

[9] Griniatsos, J., Damaskos, S., Tsekouras, N., Klonaris, C. and Georgopoulos, S. (2009) Correlation of calcified carotid plaques detected by panoramic radiograph with risk factors for stroke development. Oral Surgery Oral Medicine Oral Pathology Oral Radiology \& Endodontics, 108, 600-603. doi:10.1016/j.tripleo.2009.03.041

[10] Dolatabadi, M.A., Motamedi, M.H., Lassemi, E. and Janbaz, Y. (2010) Asymptomatic carotid artery calcifications discovered on panoramic radiograph. General Dentistry, 58, 97-99.

[11] Bayer, S., Hefgen, E.H., Bös, C., Kraus, D., Enkling, N. and Mues, S. (2011) Prevalence of findings compatible with carotid artery calcifications on dental panoramic radiographs. Clinical Oral Investigations, 15, 563-569. doi:10.1007/s00784-010-0418-6

[12] Kamikawa, R.S., Pereira, M.F., Fernandes, A. and Meurer, M.I. (2006) Study of the localization of radiopacities similar to calcified carotid atheroma by means of panoramic radiography. Oral Surgery Oral Medicine Oral Pathology Oral Radiology \& Endodontics, 101, 374-378.

\section{doi:10.1016/j.tripleo.2005.03.030}

[13] Damaskos, S., Griniatsos, J., Tsekouras, N., Georgopoulos, S., Klonaris, C., Bastounis, E. and Tsiklakis, K. (2008) Reliability of panoramic radiograph for carotid atheroma detection: A study in patients who fulfill the criteria for carotid endarterectomy. Oral Surgery Oral Medicine Oral Pathology Oral Radiology \& Endodontics, 106, 736-742. doi:10.1016/j.tripleo.2008.03.039

[14] Yoon, S.J., Yoon, W., Kim, O.S., Lee, J.S. and Kang, B.C. (2008) Diagnostic accuracy of panoramic radiography in the detection of calcified carotid artery. Dentomaxillofacial Radiology, 37, 104-108. doi:10.1259/dmfr/86909790

[15] Khosropanah, S.H., Shahidi, S.H., Bronoosh, P. and Rasekhi, A. (2009) Evaluation of carotid calcification detected using panoramic radiograph and carotid Doppler sonography in patients with and without coronary artery disease. British Dental Journal, 207, E8. doi:10.1038/sj.bdj.2009.762

[16] Spence, J.D. (2006) Technology insight: Ultrasound measurement of carotid plaque-Patient management, genetic research, and therapy evaluation. Nature Clinical Practice Neurology, 2, 611-619. doi:10.1038/ncpneuro0324

[17] Hendricson, W.D. and Cohen, P.A. (2001) Oral health care in the $21^{\text {st }}$ century: Implications for dental and medical education. Academy of Medicine, 76, 1181-1206. doi:10.1097/00001888-200112000-00009

[18] Lewis, D.A. and Brooks, S.L. (1999) Carotid artery calcification in a general dental population: A retrospective study of panoramic radiographs. General Dentistry, 47, 98-103. 